\title{
Remarks on the Donnan condenser in the sedimentation-diffusion equilibrium of charged colloids
}

\author{
Albert P Philipse \\ Van't Hoff Laboratory for Physical and Colloid Chemistry, Utrecht University, Debye Institute,
} Padualaan 8, $3584 \mathrm{CH}$ Utrecht, The Netherlands

Received 19 April 2004

Published 10 September 2004

Online at stacks.iop.org/JPhysCM/16/S4051

doi:10.1088/0953-8984/16/38/020

\begin{abstract}
The Donnan membrane equilibrium, applied to the sedimentation-diffusion (SD) equilibrium for charged colloids in gravity or an ultracentrifuge, yields limiting laws for the SD density profile and the recently predicted macroscopic electric field (van Roij 2003 J. Phys.: Condens. Matter 15 S3569) which necessarily accompanies the colloid density gradient as is also illustrated by the Donnan model. For SD equilibrium in an ultracentrifuge the radial 'Donnan' electric field accounts for the significant apparent mass reduction in the low-salt limit already discussed by Tiselius (1926 Z. Phys. Chem. 124 449). Several other aspects are discussed, such as the striking analogy between the macroscopic Donnan condenser and the usual colloidal diffuse double layer.
\end{abstract}

\section{Introduction}

The renewed interest [1-5] in the sedimentation-diffusion (SD) equilibrium of colloids, i.e. the density profile resulting from the competition between Brownian motion and gravity, has led to an intriguing insight for charged particles. Theory [4] and simulations [1,5] predict that the SD equilibrium profile of non-interacting charged colloids may deviate strongly from the 'barometric' Boltzmann exponential due to the presence of a macroscopic electric field that reduces the effective colloidal mass. Indications for such a field were reported by Biben and Hansen [1] in Monte Carlo simulations of SD profiles. These simulations were performed to explain the anomalous experimental SD profiles of Piazza et al [6] who already discussed the possible presence of an electric field that reduces the effective colloid mass. In a recent experimental study on well-defined, charged silica spheres in ethanol [3], an electric field was mentioned as one of the very few options left to explain the enormous spatial extent of SD profiles of the colloids under study, even at the very low densities where only a barometric distribution on a much smaller length scale was expected. 
Van Roij [4] has provided the first clear theoretical explanation of the electric field and its effect on SD profiles, an effect which may be drastic as confirmed by recent simulations [5]. Van Roij [4] concludes that at the level of non-interacting colloids an electric field should indeed already be present and that it causes the presence of three characteristic regions in the SD profile. The three regions, to be explained in more detail later, are region I with a 'bare' exponential profile with a decay length as if particles were uncharged, region III, which also has an exponential profile but with a decay length increased by a factor of order $z$ (the colloidal charge), and an intermediate region II where, quite surprisingly, the density profile is linear in height. A homogeneous electric field is predicted to be present in regions II and III, where the colloids are lifted upwards against gravity. In region II, it is argued [4], the effective colloid mass is nearly zero because there the electric field almost cancels the very gravity which causes its existence. Van Roij [4] derives separate expressions for the various regions from the Donnan equilibrium for non-interacting colloids, and supplements the analysis by including the electrical (Maxwell) stress in the force balance on the particles to account for the charge separation (a macroscopic condenser) responsible for the homogeneous electric field.

In this contribution we would like to point out that the Donnan equilibrium, without additional assumptions, actually yields expressions for the total SD density profile and its accompanying electric field. These expressions, which comprise the three regions referred to above, are of interest because they present a clear physical picture of the origin and effects of the electric field. Moreover, they are convenient 'limiting laws' for comparison with (and fitting of) experimental data. Briefly, for an ideal fluid of uncharged colloids one obtains the familiar barometric density profile as the equivalence of Van't Hoff's limiting law for the osmotic pressure. For charged colloids an extension beyond the Van't Hoff form is the Donnan osmotic pressure which still assumes all separate species (colloids, cations and anions) to be ideal but with the additional requirement that colloids and ions jointly satisfy electroneutrality. This Donnan pressure is equivalent to a non-barometric density profile which, consequently, solely rests on the ideal gas approximation plus electroneutrality. This is the simplest of all possible models for charged particles, which nevertheless accounts for trends in experimental data quite well [7].

The Donnan equilibrium for a homogeneous colloidal dispersion is recapitulated in section 2, and then applied to the inhomogeneous density profile in section 2.1. Since SD profiles from an analytical ultracentrifuge are important, if not indispensable, for a quantitative experimental test [7], equations are also provided for the SD equilibrium and the 'Donnan condenser' in a sector-shaped centrifugation cell. We also address the striking analogy between the macroscopic Donnan condenser and the microscopic diffuse double layer of individual colloids. Finally it is noted that the ultracentrifugation literature seems to have overlooked the importance of the inhomogeneous Donnan equilibrium, even though the early pioneers of analytical centrifugation reported on the possible effective mass reduction due to a gradient in the electrical potential.

\section{Homogeneous Donnan equilibrium}

When particles have not yet significantly settled due to gravity or a centrifugal field we have a homogeneous suspension of colloids with average number density $\bar{\rho}$, which in the Donnan model is in contact with a large reservoir with salt concentration $c_{\mathrm{s}}$ via a semipermeable membrane. The Debye length $\kappa^{-1}$ in the suspension follows from

$$
\kappa^{2}=8 \pi \lambda_{\mathrm{B}} c_{\mathrm{s}}+4 \pi \lambda_{\mathrm{B}} \bar{\rho} z
$$


where $\lambda_{\mathrm{B}}$ is the Bjerrum length (i.e. the distance at which the interaction energy between two elementary charges equals the thermal energy $k T$ ) and $z$ is the number of charges on a colloidal particle. It is convenient [4] to focus on the counter-ion concentration, instead of the colloid concentration, relative to the external salt concentration:

$$
\bar{y}=\frac{\bar{\rho} z}{2 c_{\mathrm{s}}} .
$$

In terms of this dimensionless concentration $\bar{y}$, the Debye length is

$$
\kappa^{-1}=\frac{\kappa_{0}^{-1}}{\sqrt{1+\bar{y}}},
$$

where $\kappa_{0}^{-1}$ is the Debye length in the absence of any colloids, i.e. the screening length in the salt reservoir. The Debye length measures the thickness of the diffuse ionic cloud surrounding a colloid so there is a net charge density on a length scale comparable to $\kappa^{-1}$. Thus the suspension is only electronically neutral on a length scale $L \gg \kappa^{-1}$ so the condition of electroneutrality is the volume integral

$$
\int_{\partial V} \rho^{*} \mathrm{~d} V=0 ; \quad \partial V \sim L^{3} \gg \kappa^{-3}
$$

where $\rho^{*}$ is the local charge density. For monovalent cations and anions with, respectively, concentrations $c_{+}$and $c_{-}$we can also write for negatively charged colloids

$$
\bar{\rho} z-c_{+}+c_{-}=0 \text {, }
$$

bearing in mind that this result presupposes an average over a length scale $L \gg \kappa^{-1}$. The chemical potentials of the ions are fixed by the chemical potential in the salt reservoir, for non-interacting ions given by

$$
\mu=\mu_{0}+k T \ln c_{\mathrm{s}}
$$

where $\mu_{0}$ is a standard chemical potential. For ions in the suspension, which has an electrical potential $\psi$ relative to the reservoir, an electrical term has to be added to the chemical potential:

$$
\mu_{ \pm}=\mu_{0}+k T \ln c_{ \pm} \pm e \psi
$$

in which $e$ is the elementary charge. Thus the equilibrium condition for the ions is

$$
c_{ \pm}=c_{\mathrm{s}} e^{\mp \phi} ; \quad \phi=\frac{e \psi}{k T},
$$

in which $\phi$ is the reduced Donnan potential. So the electroneutrality condition (2.5), for a suspension volume $\gg \kappa^{-3}$, yields the Donnan potential generated by the average colloid number density:

$$
\bar{y}=\frac{e^{-\phi}-e^{+\phi}}{2}=-\sinh \phi ; \quad \phi=\operatorname{arcsinh}(-\bar{y}) .
$$

In addition to the small ions, solvent molecules also equilibrate across the membrane which results in an excess osmotic pressure in the suspension:

$$
\frac{P}{k T}=\bar{\rho}+c_{+}+c_{-}-2 c_{\mathrm{s}}
$$

i.e. the well-known Donnan pressure [8-12] which is equivalent to Van't Hoff's law for noninteracting particles. Substitution of the Boltzmann ion distributions (2.8) in (2.10) yields

$$
\frac{P}{k T}=\bar{\rho}+2 c_{\mathrm{s}}(\cosh \phi-1)
$$




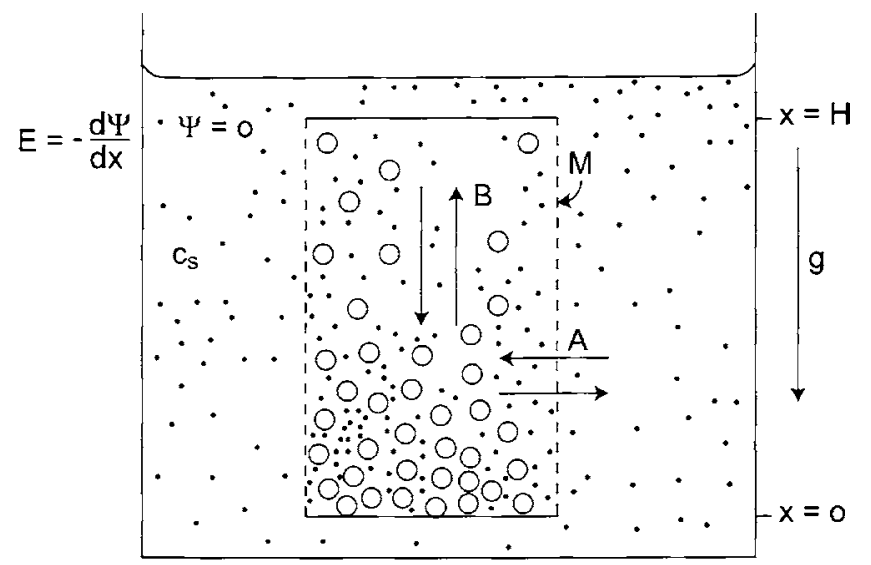

Figure 1. A schematic diagram of the inhomogeneous Donnan equilibrium: non-interacting colloids are subjected to gravity, separated from a salt reservoir with salt concentration $c_{\mathrm{s}}$ and zero electrical potential $\psi$ by a membrane M permeable only to ions and solvent. At any height $x$ there is equilibrium (A), between the solvent and ions in the reservoir and the dispersion in which the colloids adopt the sedimentation-diffusion equilibrium (B), in the sense that upward diffusion compensates the downward flux due to the net external field. The unequal distribution of charged species produces a Donnan potential $\Psi$ in the dispersion which depends on the colloid density, and therefore on $x$, resulting in the Donnan electric field $E$ which counteracts gravity.

which can be rewritten, following Van Roij's notation [4], in the dimensionless form

$$
P^{*}=\frac{P z}{2 c_{\mathrm{s}} k T}=\bar{y}+z(\cosh \phi-1) .
$$

Note that substitution of (2.9) yields the osmotic pressure either as a single-valued function of the Donnan potential:

$$
P^{*}=-\sinh \phi+z(\cosh \phi-1),
$$

or, using the identity $\cosh ^{2} \phi-\sinh ^{2} \phi=1$, as a function of the average colloid density:

$$
P^{*}=\bar{y}+z\left(\sqrt{1+\bar{y}^{2}}-1\right) \text {. }
$$

The density representation is the usual one for the Donnan pressure [8-12]. However, the $\phi$-representation equation (2.13) is instructive as it reminds us that any gradient in osmotic pressure must be accompanied by a gradient in potential, that is, an electric field. Both gradients, as explained below, therefore follow from the same force balance on the colloids.

\subsection{Inhomogeneous Donnan equilibrium}

A pressure gradient will develop in the suspension when it is subjected to gravity. In addition to the membrane equilibria ( $\mathrm{A}$ in figure 1) for ions and solvent, the charged colloids now equilibrate (B in figure 1) with the external field to produce density profiles for both colloids and ions. It should be noted that the semipermeable Donnan membrane in figure 1 is merely a conceptual device which can be replaced by any other constraint or force which keeps colloids separated from a salt reservoir. In the case of sedimentation this constraint can be gravity, separating colloids from a supernatant phase which plays the same role as the salt reservoir in a membrane equilibrium. The colloid profile follows from the isothermal force balance:

$$
\frac{\mathrm{d} P}{\mathrm{~d} x}=-\rho m g
$$


where $x$ is the vertical distance from the bottom of the suspension at $x=0$ and $m g$ is the weight of a particle with mass $m$; here and in what follows the particle weight is always corrected for buoyancy. In terms of the dimensionless pressure $P^{*}$ and dimensionless concentration $y$,

$$
\frac{\mathrm{d} P^{*}}{\mathrm{~d} x}=-\frac{y}{L} ; \quad L=\frac{k T}{m g} .
$$

Here $L$ is the gravitational length of the colloids. Integrating this differential equation for the pressure in equation (2.14), of course with $\bar{y}$ replaced by the height dependent concentration $y=y(x)$, yields

$$
\ln \frac{y}{y_{0}}+z\left[\operatorname{arcsinh} y-\operatorname{arcsinh} y_{0}\right]=\frac{-\left(x-x_{0}\right)}{L} .
$$

Here $y_{0}$ is the reduced colloid density at altitude $x_{0}$, which is an explicit function of the average density $\bar{y}$ from equation (2.2) as shown in appendix A. Since $\phi=\operatorname{arcsinh}(-y)$, equation (2.17) is equivalent to the Boltzmann distribution:

$$
y=y_{0} \exp \left[-\frac{\left(x-x_{0}\right)}{L}+z\left(\phi-\phi_{0}\right)\right],
$$

where $\phi$ and $\phi_{0}$ are the Donnan potentials at, respectively, altitude $x$ and $x_{0}$. This form confirms that the potential energy of the colloids in the inhomogeneous Donnan equilibrium is due both to gravity and an electric field. We note here the important consequence that for colloids with known gravitational length $L$, multiplying experimental concentration profiles with $\exp [x / L]$ should yield $z \phi$ as a function of height and particle concentration. Thus in principle one can directly measure Donnan potentials making use of gravity, provided that the valence $z$ of the colloids is known.

The electrical field $E$ corresponding to a particular value of $y$ is found as follows. The magnitude of the field is $E=-\mathrm{d} \psi / \mathrm{d} x$, or

$$
E^{\prime}=\frac{E e}{m g}=-L \frac{\mathrm{d} \phi}{\mathrm{d} x}
$$

where $E^{\prime}$ is a reduced field strength. The force balance (2.16) can therefore be rewritten as

$$
\frac{\mathrm{d} P^{*}}{\mathrm{~d} \phi} E^{\prime}=y
$$

which on substitution of the pressure in (2.13) yields the field strength:

$$
E^{\prime}=\frac{-y}{z y+\sqrt{1+y^{2}}} .
$$

Since this field is the gradient of the Donnan potential it is logical to call it the Donnan electric field. Three regimes can be distinguished in this Donnan field:

$$
E^{\prime} \sim\left\{\begin{array}{lll}
-1 /(1+z), & y \gg 1 \\
-1 / z, & y \ll 1 \text { and } z y \gg 1 & \text { III, } \\
-y, & y \ll 1 \text { and } z y \ll 1 & \text { II, }
\end{array}\right.
$$

The field is homogeneous in the first two regions, where the charge $z$ has a significant effect on the colloid profile; the field vanishes as $E^{\prime} \sim y \sim \exp [-x / L]$ in region I where the external salt dominates the counter-ions. Making use of the logarithmic representation [13]

$$
\operatorname{arcsinh} y=\ln \left[y+\sqrt{1+y^{2}}\right],
$$


the density profiles for the three regions are found from equation (2.17) to be, apart from integration constants,

$y \sim \begin{cases}\exp [-x /(1+z) L], & y \gg 1 \\ -x / z L, & y \ll 1 \quad \text { and } z y \gg 1 \\ \exp [-x / L], & y \ll 1 \quad \text { and } z y \ll 1\end{cases}$

which are precisely the three regions identified by Van Roij [4]. For further interpretation of the inflated gravitational length $(1+z) L$ in region III, see appendix B. The foregoing results for the inhomogeneous Donnan equilibrium in the gravity field are easily extended to colloids in a centrifuge. The main difference is that now the acceleration changes in the radial direction, producing a radial Donnan electric field. For a colloidal dispersion which rotates at an angular frequency $\omega$ the centripetal acceleration at a distance $r$ from the rotation axis at $r=0$ equals $\omega^{2} r$. Thus the force balance for the colloids is

$$
\frac{\mathrm{d} P}{\mathrm{~d} r}=-\rho m \omega^{2} r
$$

with $m$ the colloid mass, again corrected for buoyancy. In terms of dimensionless pressure in equation (2.12) we have

$$
\frac{\mathrm{d} P^{*}}{\mathrm{~d} r}=-\frac{y r}{\lambda^{2}} ; \quad \lambda=\sqrt{\frac{k T}{m \omega^{2}}} .
$$

Here $\lambda$ can be identified as a 'centrifugal length', in analogy with the gravitational length $L$. Substitution of equation (2.14) and integration yields

$$
\ln \frac{y}{y_{0}}+z\left[\operatorname{arcsinh} y-\operatorname{arcsinh} y_{0}\right]=\frac{-\left(r^{2}-r_{0}^{2}\right)}{2 \lambda^{2}}
$$

in which $y_{0}$ is the reduced colloid density at a distance $r_{0}$ from the rotation axis. The three regimes in the density profile adopt, instead of (2.24), the form

$$
y \sim \begin{cases}\exp \left[-r^{2} / 2(1+z) \lambda^{2}\right] & \text { III, } \\ -r^{2} / 2 z \lambda^{2} & \text { II, } \\ \exp \left[-r^{2} / 2 \lambda^{2}\right] & \text { I. }\end{cases}
$$

The peculiar regime II in a rotating dispersion is now part of a parabola, instead of the linear function in the gravity field. The Donnan electric field $E=-\mathrm{d} \psi / \mathrm{d} r$ is now competing with a colloid weight $m \omega^{2} r$ instead of $m g$. Thus the dimensionless field strength is

$$
E^{\prime}=\frac{E e}{m \omega^{2} r}=-\frac{\lambda^{2}}{r} \frac{\mathrm{d} \phi}{\mathrm{d} r}
$$

which can be used to rewrite the centrifugal force balance equation (2.25) as

$$
\frac{\mathrm{d} P^{*}}{\mathrm{~d} \phi} E^{\prime}=y
$$

a result identical to equation (2.20) for the gravitational force balance. Consequently the Donnan field in a rotating colloidal dispersion has the same dimensionless form as in equations (2.21) and (2.22) for the gravitational SD equilibrium. The absolute field strength in the three regions is proportional to

$$
E e \sim \begin{cases}-m \omega^{2} r /(1+z) & \text { III, } \\ -m \omega^{2} r / z & \text { II, } \\ -m \omega^{2} r \exp \left[-r^{2} / 2 \lambda^{2}\right] & \text { I. }\end{cases}
$$

The physical meaning is clear: at $r=0$ there is no centripetal acceleration and therefore no Donnan field, whereas at increasing $r$ the apparent colloid weight increases which enhances the colloid-counter-ion charge separation and therefore the magnitude of the electric field. 


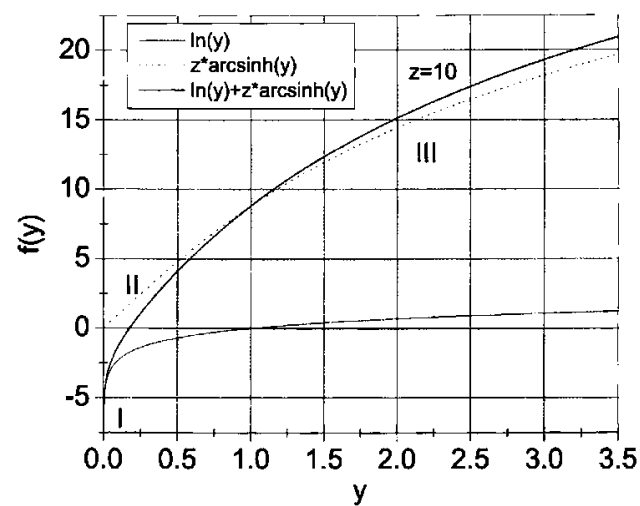

Figure 2. A plot of the barometric $(\ln y)$ part and the condenser part $(z \operatorname{arcsinh} y)$ for $z=10$, with an indication for the three regions in the density profile distinguished in equations (2.22) and (2.23). The parameter $y$ is the ratio of counter-ions to external salt ions. Note that even for this moderate $z$, the condenser term dominates the larger part of the profile, though region II is narrow.

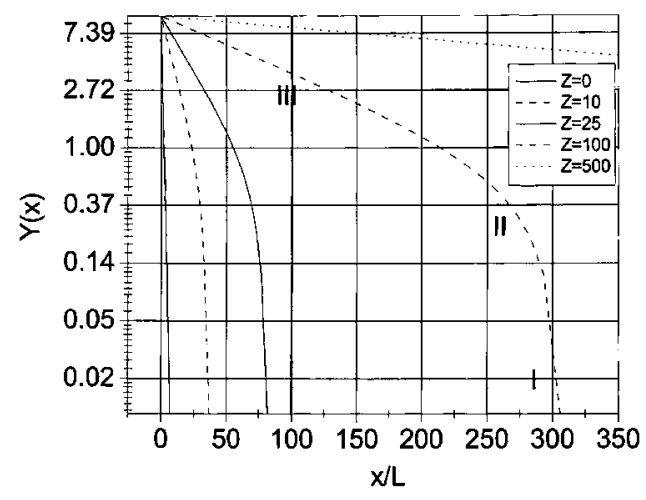

Figure 3. Colloid density profiles according to equation (2.17) for various values of the colloidal charge $z$. Note the strong inflation of the profiles in comparison to the Boltzmann profile for uncharged particles $(z=0)$.

\section{Discussion}

Expression (2.17) for the colloid density profile clearly identifies the two contributions to the sedimentation-diffusion equilibrium within the Donnan approximation, namely the purely logarithmic term (the barometric term) which would remain for non-charged particles and the $z$ dependent inverse hyperbolic term (the condenser term) which stems from the charge $z$ on the colloids (figures 2, 3). So charged colloids, as is also clear from [4], never exactly adopt a barometric distribution, even if the colloids do not interact throughout the whole density profile. At sufficiently high altitude the profile tends asymptotically to the barometric distribution because at some point the colloid-counter-ion densities are so low that the external salt dominates, effectively removing the $z$ dependent term (though the field in region I is not zero; see equation (2.22)). The whole profile can be seen as a fairly thin barometric distribution that 'floats' on top of a $z$ dependent condenser.

The condenser part has two interesting regions which follow from the asymptotes of the inverse hyperbolic sine in equation (2.23), namely an exponential part (region III), with an expanded gravitational length $(1+z) L$, and region II, with a linear concentration decay. The peculiar linear region, first identified by van Roij [4], is, as he points out, equivalent to a purely quadratic equation of state. Indeed, substitution of $P \sim y^{2}$ in the force balance (2.16) produces a linear sedimentation-diffusion equilibrium profile. Van Roij also noted that in this region the electric field nearly cancels the effect of gravity, which captures the essence of the inhomogeneous Donnan equilibrium: the weight of the colloids is almost compensated by their own charge, producing a reduction in effective mass which strongly inflates a sedimentationdiffusion equilibrium profile. One can also say that for (non-interacting) uncharged colloids the SD profile measures the colloid mass, whereas for colloids with total charge $q$ the profile depends on the charge-to-mass ratio, namely

$$
z L=\frac{q}{m} \frac{k T}{e g} .
$$


The density profile equation (2.17) has been obtained here from the force balance equation (2.15). Since the profile is an equilibrium distribution the total chemical potential of the colloids, $\tilde{\mu}$, must be independent of height:

$$
\frac{\partial}{\partial x} \tilde{\mu}=0 \text {. }
$$

In the chemical potential of the ions in (2.7) their weight does not appear, but for the much heavier colloids, of course, we have to include their potential energy in the gravity field. Hence for a negatively charged, ideal colloid,

$$
\tilde{\mu}=\mu_{0}+k T \ln \rho-z e \psi+m g x .
$$

Substitution in the equilibrium condition (3.2) yields, after rewriting to dimensionless variables,

$$
\ln y-z \phi=C-\frac{x}{L} \text {. }
$$

Taking this together with the equilibrium distributions for the two ionic species in (2.8), and the electroneutrality condition (2.5), we have four equations which determine four unknowns, namely the gradient in electrical potential and the gradients in concentrations of colloids, anions and cations. The results are exactly the same as those obtained in section 2.1 from the Donnan osmotic pressure because they rest on the same assumptions, namely ideal behaviour plus charge neutrality.

What about the compatibility of the condenser producing the electric field with Donnan's electroneutrality condition? Van Roij [4] concludes that sometimes the charges of the condenser are located in a small layer with thickness of the order of the Debye length $\kappa^{-1}$. First it should be noted that the very existence of charge separation is not inconsistent with Donnan's model. Such separation is a necessary consequence of the inhomogeneous Donnan equilibrium: distributing particles implies distributing the electrical potential so there must be an electric field and a corresponding (diffuse) condenser. However, the Donnan model cannot specify with the desired spatial resolution where charges are located, because for the electroneutrality condition, equation (2.4), the suspension has been coarse grained into volumes $\gg \kappa^{-3}$ with a certain average electrical potential, disregarding all details of the electric fields in these volumes. Thus the fact that the condenser charges are located in a region with thickness $\kappa^{-1}$ does not follow from the Donnan model but does not contradict it either, just as the diffuse double layer around a single colloids - also charge separation on a scale $\kappa^{-1}$-is compatible with, though certainly not a consequence of, Donnan's electroneutrality.

The analogy between the 'Donnan condenser' and the microscopic electrical double layer surrounding a colloid can be extended further: the Donnan condenser clearly has features of a macroscopic diffuse double layer. For example, it shrinks when salt is added and expands upon deionization. This salt effect can be easily understood from figure 2: if in the initial homogeneous suspension the ionic strength is lowered at fixed total colloid concentration, then $\bar{y}=\bar{\rho} z / 2 c_{\mathrm{s}}$ shifts in figure 2 to the right, and thus the whole profile expands. Also from the condenser viewpoint there is a similarity. From classical electrochemistry [11] it is well known that the microscopic (Gouy-Chapman) double layer resembles a flat condenser, which for small surface potentials obeys [11]

$$
\sigma=C \psi_{0} ; \quad C=\frac{\varepsilon}{4 \pi} \frac{1}{\kappa^{-1}}
$$

Here $\sigma$ is the charge density, $\psi_{0}$ the colloid surface potential and $\kappa^{-1}$ can be interpreted as the distance between the two plates of a condenser with capacitance $C$. The sedimentationdiffusion profile can — be it very qualitatively_-be seen as a huge series connection of such 
colloidal condensers. In that case the total capacitance is

$$
\frac{1}{C_{\mathrm{tot}}}=\sum_{i=1}^{N} \frac{1}{C_{i}}=\frac{4 \pi}{\varepsilon} \sum_{i=1}^{N} \kappa_{i}^{-1},
$$

where $\kappa_{i}^{-1}$ is the thickness of capacitor $i$. The length scale for the SD profile is of order $(z+1) L$; therefore the number of capacitors in the series is of order $N \sim(z+1) L / \kappa_{0}^{-1}$, if we take all screening lengths equal to $\kappa_{0}^{-1}$ (see, however, equation (2.3)). Hence for the Donnan condenser we find

$$
\sigma=C_{\text {tot }} \psi_{0} ; \quad C_{\text {tot }} \sim \frac{\varepsilon}{4 \pi} \frac{1}{(z+1) L},
$$

which indeed confirms that the Donnan condenser is quite similar to a colloidal condenser, only with a much smaller capacitance, because the condenser 'plates' have been separated to a very large distance. For a series connection the total potential jump remains, at least in order of magnitude, the same as in the colloidal double layer. For the Donnan condenser the potential difference between the plates is $\psi_{0} \sim E(z+1) L$ which on substitution of (2.22) yields $\psi_{0} \sim-k T / e=25 \mathrm{mV}$. This is also a typical value for the potential drop around an individual colloid. So the much smaller capacitance of the Donnan condenser leads to very low charge densities on its 'plates'. However, as noted by van Roij [4], it takes only very little excess charge to lift up colloids against gravity.

Since the Donnan equilibrium and sedimentation are both classical topics of physical chemistry, one wonders whether their combination has not been investigated earlier. Donnan himself [8, 9] does not mention the issue. Tiselius [14], however, noted that a protein concentration gradient will give rise to a Donnan potential gradient which opposes the centrifugal field. He also derived the low-salt limit (region III) in equation (2.28). Bolam [10] observed that the reduction of the effective mass with a factor $z+1$ in Tiselius's formula is a maximum effect for very low salt concentration and that the potential gradient should be practically eliminated at high ionic strength. No attempts were made, however, to quantify colloid profiles as a function of salt concentration or to further investigate or quantify the electric field. Neither were such attempts made by Svedberg and Pederson in their classical book [15], though they refer to Tiselius and his derivation of the $1+z$ mass reduction. Later reviews of ultracentrifugation and colloid textbooks briefly mention Tiselius's result $[16,17]$ or omit the topic $[11,18,19]$. In the modern (largely biochemical) ultracentrifugation literature [20], only very occasionally is reference made to the 'Donnan effect' in the sedimentation equilibrium, be it only a reference to gradients in salt and polyelectrolyte concentration, and not to the accompanying gradient in electrical potential [21]. So it seems that the explicit notion of a potential gradient has somehow disappeared in the literature on the sedimentation equilibrium of polyelectrolytes. In this respect it is interesting to note that the proof of the monodispersity of haemoglobin molecules by Svedberg is an often quoted starting point for quantitative ultracentrifugation. However, the same protein was used by Nichols [10, 22], in a study almost never quoted, to estimate the effect of an electrical potential gradient by comparing measured density profiles to calculations based on a molecular weight $M=68500 \mathrm{~g} \mathrm{~mol}^{-1}$ of haemoglobin. Even if the calculations are redone with the correct molecular mass [23] of $M=64500 \mathrm{~g} \mathrm{~mol}^{-1}$, a significant discrepancy with Nichols' measurements remains, which very likely is due to the Donnan electric field.

\section{Conclusions and outlook}

The sedimentation-diffusion (SD) equilibrium of charged colloids, and the accompanying macroscopic electric field in a homogeneous or radial centrifugal field, can be treated 
analytically within the Donnan approximation, i.e. for non-interacting colloids and ions which together only have to satisfy electroneutrality. The formula for the colloid density profile yields the correct low-salt limit, a strongly inflated exponent already identified by Tiselius [12], the familiar barometric exponential at high ionic strength and the peculiar intermediate linear density profile found by van Roij [4]. The electric field is a necessary consequence of an inhomogeneous Donnan equilibrium, though within the Donnan approximation it is not possible to indicate where the 'condenser charges' are located with the required resolution on the length scale of the Debye screening length. The 'Donnan condenser', which in the gravity field can even be on the centimetre scale, resembles a huge series connection of colloidal capacitors and can be seen as a macroscopic consequence of microscopic electrical double layers.

The density profile equation (2.17) may be useful in practice for estimating the colloid charge $z$ by fitting an experimental density profile. Particularly intriguing is the possibility of extracting Donnan potentials directly from the profile as mentioned below equation (2.18). Since within the Donnan approximation the colloid size and shape are irrelevant, equation (2.17) may apply to inorganic colloidal spheres, rods or (clay) platelets, as well as charged biomolecules. In particular, at a $\mathrm{pH}$ far away from the isoelectric point the contribution of counter-ions may be large enough to probe the whole profile equation (2.17) at concentrations low enough to satisfy at all altitudes the ideal gas approximation. Clearly the reported routine practice [16] for determining molecular weights of polyelectrolytes swamped with salt suppresses information on the charge-to-mass ratio of the macromolecules under study. Finally, it should be noted that the Donnan equilibrium in its simplest form may be a poor description of charged colloids in the more concentrated parts of a sedimentation profile. In that case equation (2.17) is anyway useful for assessing the presence of non-idealities in experimental density profiles, and for providing a starting point for further theoretical analysis beyond the Donnan approximation.

\section{Acknowledgments}

M Rasa, R van Roij, G Koenderink and B Erné are thanked for stimulating discussions and fruitful collaboration. M Rasa is also thanked for a critical reading of the manuscript. H Lekkerkerker and A Vrij are acknowledged for their thoughtful comments on the Donnan equilibrium. This work is part of a project financially supported by the Netherlands Organization for Scientific Research (NWO, Stichting Chemische Wetenschappen).

\section{Appendix A. Integration constants}

The integration constant in (2.17) follows from mass conservation:

$$
\int_{x_{0}}^{\infty} \rho(x) \mathrm{d} x=\frac{N_{\text {tot }}}{A} .
$$

Here $N_{\text {tot }}$ is the total number of colloids above a certain altitude $x_{0}$ in a column with cross sectional area $A$. The upper limit of the integral, actually the height $H$ of the vessel, is taken to be infinite, assuming that $H$ is large enough for the colloid density $\rho(x)$ to completely decay, i.e. $H \gg z L$. In terms of the dimensionless concentration $y$,

$$
\int_{x_{0}}^{\infty} y \mathrm{~d} x=\frac{N_{\text {tot }}}{A} \frac{z}{2 c_{\mathrm{s}}}=\bar{y} H,
$$


in which $\bar{y}$ is average density, or the density defined in (2.2) of the initially homogeneous suspension in a vessel with height $H$. The density profile (2.17) is the solution of

$$
\frac{1}{y} \mathrm{~d} y+z \frac{\mathrm{d} y}{\sqrt{1+y^{2}}}=-\frac{\mathrm{d} x}{L} .
$$

Taking $y_{0}$ to be the concentration at $x_{0}$ it follows that

$$
\frac{1}{L} \int_{x_{0}}^{\infty} y \mathrm{~d} x=-\int_{y_{0}}^{0} \mathrm{~d} y-z \int_{y_{0}}^{0} \frac{y \mathrm{~d} y}{\sqrt{1+y^{2}}}=y_{0}-z+z \sqrt{1+y_{0}^{2}} .
$$

Thus the full density profile is

$$
\ln \frac{y}{y_{0}}+z\left[\operatorname{arcsinh} y-\operatorname{arcsinh} y_{0}\right]=\frac{-\left(x-x_{0}\right)}{L},
$$

where $y_{0}$ is, according to (A.2) and (A.4), determined by

$$
y_{0}-z+z \sqrt{1+y_{0}^{2}}=\frac{\bar{y} H}{L}
$$

with the solutions

$$
y_{0}= \begin{cases}\frac{\bar{y} H}{L}, & z=0 \\ \frac{\left(\frac{\bar{y} H}{L}+1\right)^{2}-1}{2\left(\frac{\bar{y} H}{L}+1\right)}, & z=1 \\ \frac{\beta}{z^{2}-1} \sqrt{1-\frac{\left(z^{2}-1\right)\left(z^{2}-\beta^{2}\right)}{\beta^{2}}}-\frac{\beta}{z^{2}-1}, & z>1\end{cases}
$$

where $\beta=\frac{\bar{y} H}{L}+z$. For highly charged colloids $(z \gg 1)$ in a sufficiently large vessel,

$$
y_{0} \sim \frac{\bar{y} H}{z L}, \quad \text { for } H \gg \frac{z L}{\bar{y}} .
$$

Here $y_{0} \gg 1$, so $x_{0}$ is located in region III. The limiting result (A.8), of course, also directly follows from (A.6) taking $y_{0} \gg 1$. The value of $y_{0} \sim \bar{y} H / L$ for the case $x_{0}$ is located in the barometric region and follows from the limit $z \rightarrow 0$ in equation (A.6). For $x_{0}=0, y_{0}$ is the concentration at the bottom of the profile and $\bar{y}$ is the average for the total initial suspension. If for this suspension the external salt strength $c_{\mathrm{s}}$ and the average colloid number density $\bar{\rho}$ are also known (see equation (2.2)), then the colloid charge $z$ is the only free parameter left for a fit of experimental data to (A.5).

\section{Appendix B. Polydispersity}

The significant reduction of the effective colloid mass by the term $1+z$ in region III can also be interpreted as a polydispersity effect. Consider an ideal mixture of $j=0,1, \ldots, N$ species of uncharged particles which produce a sum of independent force balances as in (2.15):

$$
\sum_{j=0}^{N} \frac{\mathrm{d} P_{j}}{\mathrm{~d} x}=-\sum_{j=0}^{N} \rho_{j} m_{j} g ; \quad P_{j}=\rho_{j} k T .
$$

Integration yields the exponential distribution of the total particle number density:

$$
\sum_{j=0}^{N} \rho_{j}(x)=\sum_{j=0}^{N} \rho_{j}\left(x_{0}\right) \exp \left[-\frac{\left(x-x_{0}\right)}{\langle L\rangle}\right],
$$


with a gravitational length determined by the number-averaged particle mass:

$$
\langle L\rangle=\frac{k T}{\langle m\rangle g},
$$

where the brackets denote an average over the size distribution. For a two-component mixture of large (l) and small (s) particles,

$$
\langle L\rangle=\frac{(1+\alpha) k T}{\left(m_{1}+\alpha m_{\mathrm{s}}\right) g} \sim \frac{(1+\alpha) k T}{m_{1} g} .
$$

Here $\alpha$ is the ratio of the total numbers of small and large particles. Thus the inflation of the average gravitational length with a factor $1+\alpha$ is simply due to the almost weightless, small particles added to the large colloids. The Donnan osmotic pressure increases with the same factor and the reduced mass of the large particles (the colloids) obtained from this pressure is $m_{l} / 1+\alpha$. However, to obtain this reduced mass from sedimentation, equation (B.2) is inconvenient, as one measures only the density profile of the large particles (colloids) and not the total number densities as required by (B.2). Now suppose that each large particle is always accompanied by the same number of small satellite particles. Then the concentration ratio is independent of altitude $x$, in which case

$$
\rho_{L}(x)+\rho_{\mathrm{s}}(x)=\rho_{L}(x)(1+\alpha),
$$

with the result that (B.2) for the two-component mixture simplifies to the colloid density profile:

$$
\rho_{L}(x)=\rho_{L}\left(x_{0}\right) \exp \left[-\frac{x}{(1+\alpha) L_{1}}\right] .
$$

Of course, this is region III in (2.24) and (B.5) is Donnan's electroneutrality condition (2.5) for charged colloids and their $\alpha$ counter-ions in the limit of zero external salt.

\section{References}

[1] Biben T and Hansen J P 1994 J. Phys.: Condens. Matter A 6345

[2] Löwen H 1998 J. Phys.: Condens. Matter 10 L479

[3] Philipse A P and Koenderink G H 2003 Adv. Colloid Interface Sci. 100-102 603

[4] van Roij R 2003 J. Phys.: Condens. Matter 15 S3569

[5] Hynninen A P, van Roij R and Dijkstra M 2004 Europhys. Lett. 65719

[6] Piazza R, Bellini T and Degiorgio V 1993 Phys. Rev. Lett. 714267

[7] Rasa M and Philipse A P 2004 Nature 429857

[8] Donnan F G and Harris A B 1911 J. Chem. Soc. 991554

[9] Donnan F G 1911 Z. Electrochem. 17572

Donnan F G 1924 Chem. Rev. 173

[10] Bolam T R 1932 The Donnan Equilibria and their Application to Chemical, Physiological and Technical Processes (London: G Bell and Sons)

[11] Overbeek J Th G 1952 Colloid Science vol 1, ed H R Kruyt (Amsterdam: Elsevier)

[12] Mysels K J 1959 Introduction to Colloid Chemistry (New York: Interscience)

[13] Abramowitz M and Stegun I A (ed) 1972 Handbook of Mathematical Functions (New York: Dover)

[14] Tiselius A 1926 Z. Phys. Chem. 124449

[15] Svedberg Th and Pederson O 1940 Die Ultrazentrifuge (Dresden: Steinkopf)

[16] Schachmann H K 1959 Ultracentrifugation in Biochemistry (New York: Academic)

[17] Alexander A E and Johnson P 1949 Colloid Science vol 1 (Oxford: Clarendon)

[18] Williams J W 1963 Ultracentrifugal Analysis in Theory and Experiment (New York: Academic)

[19] van Holde K E 1971 Physical Biochemistry (Englewood Cliffs, NJ: Prentice-Hall)

[20] Harding S E, Rowe A J and Norton J C (ed) 1992 Analytical Ultracentrifugation in Biochemistry and Polymer Science (Cambridge: The Royal Society of Chemistry)

[21] Budd P M 1992 Analytical Ultracentrifugation in Biochemistry and Polymer Science ed S E Harding, A J Rowe and J C Norton (Cambridge: The Royal Society of Chemistry)

[22] Nichols J B 1930 J. Am. Chem. Soc. 525176

[23] Lehninger A L 1978 Biochemistry (New York: Worth Publishers) 